\title{
POINTWISE CHARACTERIZATIONS OF HARDY-SOBOLEV FUNCTIONS
}

\author{
Pekka Koskela and Eero Saksman
}

\begin{abstract}
We establish pointwise characterizations of functions in the Hardy-Sobolev spaces $H^{1, p}$ within the range $p \in(n /(n+1), 1]$. In particular, a locally integrable function $u$ belongs to $H^{1, p}\left(\mathbf{R}^{n}\right)$ if and only if $u \in L^{p}\left(\mathbf{R}^{n}\right)$ and it satisfies the Hajłasz type condition

$$
|u(x)-u(y)| \leq|x-y|(h(x)+h(y)), \quad x, y \in \mathbf{R}^{n} \backslash E,
$$
\end{abstract}

where $E$ is a set of measure zero and $h \in L^{p}\left(\mathbf{R}^{n}\right)$. We also investigate Hardy-Sobolev spaces on subdomains and extend Hardy inequalities to the case $p \leq 1$.

\section{Introduction}

It is a well-established fact that, for the purposes of harmonic analysis or theory of partial differential equations, the right substitute for $L^{p}\left(\mathbf{R}^{n}\right)$ in case $p \in(0,1]$ is the (real) Hardy space $H^{p}\left(\mathbf{R}^{n}\right)$, or it's local version $h^{p}\left(\mathbf{R}^{n}\right)$. These spaces behave nicely under the action of regular singular integrals or pseudo-differential operators. Moreover, for the Hardy spaces the Paley-Littlewood theory and interpolation results extend to the whole scale of Lebesgue exponents $p \in(0, \infty)$. It is hence natural to investigate Sobolev spaces where one demands that derivatives up to a fixed order belong to a Hardy type space with $p \leq 1$. After the fundamental work of Fefferman and Stein [8] this line of research was initiated by Peetre in early 70's, and it was generalized and carried further by Triebel and others. We refer to [26], [30] for extensive accounts on general Besov and Triebel -type scales of function spaces in the case $p \in(0,1]$.

In this paper we obtain new pointwise characterizations of Hardy-Sobolev spaces in the most important case where the smoothness index is one and the elements in these spaces are honest functions, i.e. they belong to $L_{l o c}^{1}$. Recall that a distribution $f \in S^{\prime}\left(\mathbf{R}^{n}\right)$ belongs to the homogeneous (Hardy-)Sobolev space $\dot{H}^{1, p}\left(\mathbf{R}^{n}\right)$ if $D_{k} f \in H^{p}\left(\mathbf{R}^{n}\right)$ for $1 \leq k \leq n$. Modulo polynomials, these spaces coincide with the homogeneous spaces considered in [30, Chapter 5]. Various characterizations in terms of Paley-Littlewood decomposition (the square function), Lusin functions, atoms, maximal operators, or various integral means are contained e.g. in [30] and more recents books by the same author.

Received by the editors February 28, 2007.

2000 Mathematics Subject Classification. Primary 46E35, 42B30, secondary 26D15, 42B25.

Key words and phrases. Hajłasz-Sobolev spaces, Hardy-Sobolev spaces, pointwise inequalities, Hardy inequalities.

The first author was supported by the Academy of Finland, project 120972. The second author was supported by the Academy of Finland, projects no. 113826 and 118765, and by the Finnish Center of Exellence Analysis and Dynamics. 
Strichartz [28] found (see also [5]) equivalent norms for $\dot{H}^{1, p}\left(\mathbf{R}^{n}\right)$ or, more generally, for corresponding spaces with fractional smoothness and Lebesgue exponents in the range $p>n /(n+1)$. In this case the elements in spaces $\dot{H}^{1, p}\left(\mathbf{R}^{n}\right)$ are locally integrable. Thus, $\|f\|_{\dot{H}^{1, p}} \sim\left\|D_{2,1} f\right\|_{p}$, where $p>n / n+1$, and

$$
D_{2,1}(f)(x)=\left(\int_{0}^{\infty}\left[\int_{B(0,1)}\left|\Delta_{r y}^{2} f(x) d y\right|\right]^{2} r^{-3} d r\right)^{1 / 2} .
$$

Above $\Delta_{t}^{2} f(x)=f(x+2 t)-2 f(x+t)+f(x)$, whence Strichartz's characterization is pointwise, but it employs integrated second differences.

Closer to our results is the elegant work of Miyachi [24] where counterparts of previous results of Calderón (and of the general theory of DeVore and Sharpley [7]) are obtained in the case of the Hardy-Sobolev spaces in terms of maximal functions related to mean oscillation of the function in cubes. Especially, Miyachi showed that for $p \in(n /(n+1), 1]$ we have that $f \in \dot{H}^{1, p}$ if and only if $g \in L^{p}$ where

$$
g(x):=\sup _{r>0} r^{-1} f_{B(x, r)}\left|f-f_{B(x, r)}\right| d x .
$$

Also the papers of Gatto, Segovia, and Jimenez [9], Janson [18] and Orobitg [25] are related to the theme of the present paper. More recently there has been a renewed interest in Hardy-Sobolev spaces $H^{1, p}$ and their variants on $\mathbf{R}^{n}$, or on subdomains. Chang, Dafni, and Stein [4] (see also [3]) consider Hardy-Sobolev spaces in connection with estimates for elliptic operators, whereas Aucher, Emmanuel, and Tchamitchian [2] study these spaces with applications to square roots of elliptic operators.

Our main result shows that there is a surprisingly simple strictly pointwise characterization of the homogeneous (Hardy-)Sobolev space simply in terms of first differences:

Theorem 1. Let $n \geq 1$ and $\frac{n}{n+1}<p \leq 1$. Then a distribution $f \in S^{\prime}\left(\mathbf{R}^{n}\right)$ belongs to $\dot{H}^{1, p}\left(\mathbf{R}^{n}\right)$ if and only if $f$ is locally integrable and there is a function $g \in L^{p}$ such that

$$
|f(x)-f(y)| \leq|x-y|(g(x)+g(y)), \quad x, y \in \mathbf{R}^{n} \backslash E
$$

where $E$ is a set of measure zero. Moreover, one has the equivalence of (quasi)norms

$$
\|f\|_{\dot{H}^{1, p}} \sim \inf \|g\|_{p}
$$

where the infimum is taken over all admissible functions $g$ in (1), and one identifies functions differing only by a constant.

It was previously known that the above characterization holds true in the case $p>$ 1. In [11], Hajłasz proposed to use (1) as a definition of Sobolev-spaces on arbitrary metric spaces. A considerable activity (see e.g. [11], [16], [13] and the references therein) has been devoted to the study of the corresponding (non-homogeneous) spaces $M^{1, p}$, as they are customarily denoted. In the case $p>n /(n+1)$ we may define the non-homogeneus Sobolev spaces $H^{1, p}\left(\mathbf{R}^{n}\right)$ by adding to the definition of $\dot{H}^{1, p}\left(\mathbf{R}^{n}\right)$ the condition $f \in L^{p}\left(\mathbf{R}^{n}\right)$. When our result is combined with the previously known case $p>1$ (recall that $L^{p}\left(\mathbf{R}^{n}\right)=H^{p}\left(\mathbf{R}^{n}\right)$ if $p>1$ ), we obtain the norm equivalence

$$
\|f\|_{H^{1, p}\left(\mathbf{R}^{n}\right)} \sim\|f\|_{M^{1, p}\left(\mathbf{R}^{n}\right)} \text { for } p>\frac{n}{n+1},
$$


which, incidentally, solves the characterization problem of $M^{1,1}\left(\mathbf{R}^{n}\right)$ that has been open after [11]. This fact also testifies for the naturality of the spaces of type $M^{1, p}$ in the case of Euclidean spaces: they yield the right spaces in view of harmonic analysis also in the case $p \leq 1$.

For the precise definitions of the Sobolev and Hardy spaces we refer to Section 2, which also contains auxiliary results and the proof of Theorem 1 . The remaining two sections provide examples of the flexibility and strength of Theorem 1. In the present paper we aim to concentrate on key ideas, whence we have not striven here for most general results.

More specifically, Section 3 starts by treating the case where the derivative belongs to a local Hardy space. In addition, pointwise characterizations analoguous to Theorem 1 are given for the spaces $H^{1, p}(\Omega)$ defined on subdomains $\Omega \subset \mathbf{R}^{n}$. These results are obtained by slight modifications of the considerations of Section 2. Moreover, we scetch a transparent proof of Jones-Miyachi's extension result for uniform domains.

Section 4 in turn applies our characterization to extend effortlessly the classical Hardy inequality also to case $p \leq 1$ in the framework of Hardy-Sobolev spaces. This is a novel range of exponents, since it is well known that e.g. for the space $W^{1,1}(\Omega)$ the Hardy inequality is not true even if the domain $\Omega$ is a ball.

\section{Definitions, auxiliary results and proof of Theorem 1}

We begin by shortly recalling the relevant definitions and results from the theory of real Hardy spaces. For the readers convenience we use as a principal reference the monograph [27]. Fix a compactly supported function $\psi \in C_{0}^{\infty}\left(\mathbf{R}^{n}\right)$ with $\int \psi=1$ and with supp $\psi \subset\{x:|x| \leq 1\}$. Assume that $p>0$ and consider $f \in \mathcal{S}^{\prime}\left(\mathbf{R}^{n}\right)$, i.e. $f$ is a tempered distribution. By the definition of Fefferman and Stein, $f$ belongs to $H^{p}\left(\mathbf{R}^{n}\right)$ if and only if $\mathcal{M}_{\psi} f \in L^{p}\left(\mathbf{R}^{n}\right)$, where

$$
\mathcal{M}_{\psi} f(x):=\sup _{t>0}\left|f * \psi_{t}(x)\right| .
$$

Above $\psi_{t}(y)=t^{-n} \psi(y / t)$. The corresponding norm (quasi-norm in case $p<1$ ) is obtained by setting $\|f\|_{H^{p}}:=\left\|\mathcal{M}_{\psi} f\right\|_{L^{p}}$. One may replace $\psi$ by any element in $\mathcal{S}\left(\mathbf{R}^{n}\right)$ and obtain an equivalent norm. In what follows we shall denote by $M f$ the standard Hardy-Littlewood maximal function of $f$.

We also recall a local version of the so-called grand maximal function. The basic significance of the grand maximal function comes from the fact that it allows one to use certain controlled families of test functions in the maximal function, instead of a single one. For any radius $r>0$, natural number $N \geq 1$, and any distribution $f \in \mathcal{D}^{\prime}(B(x, r))$, we define

$$
\mathcal{M}_{r, N} f(x)=\sup _{\substack{\varphi \in C_{0, N}^{\infty}\left(B\left(x, r^{\prime}\right)\right) \\ 0<r^{\prime} \leq r}}|\langle f, \varphi\rangle|,
$$

and the class of functions $\varphi$ over which the supremum is taken is

$$
C_{0, N}^{\infty}(B)=\left\{\varphi \in C_{0}^{\infty}(B(x, r)):\left|\partial^{\alpha} \varphi\right| \leq r^{-n-|\alpha|} \text { for }|\alpha| \leq N\right\} .
$$

Above $\langle f, \varphi\rangle$ stands for the action of the distribution $f$ on the test function $\varphi$. Observe that if $\varphi \in C_{0, N}^{\infty}(B)$ then for any $t>0$ one has $t^{-n} \varphi\left(t^{-1} \cdot\right) \in C_{0, N}^{\infty}(t B)$. If the supremum in (3) is taken over all positive radii, that is if $r=\infty$, we use the shorthand $\mathcal{M}_{N} f(x):=\mathcal{M}_{\infty, N} f(x)$. Assuming that $N$ is large enough (depending on $p, n$, see $[27$, 
III 1.8, 5.9]), a distribution $f \in \mathcal{S}^{\prime}\left(\mathbf{R}^{n}\right)$ belongs to $H^{p}\left(\mathbf{R}^{n}\right)$ if and only if $\mathcal{M}_{N} f(x) \in$ $L^{p}\left(\mathbf{R}^{n}\right)$. In the range $p>n /(n+1)$ we may choose $N=1$. For vector valued functions $f=\left(f_{1}, \ldots, f_{n}\right)$ we set $\mathcal{M}_{r, N} f(x)=\max _{1 \leq j \leq n} \mathcal{M}_{r, N} f_{j}(x)$.

Recall that, in the case $p>1$, the homogeneous Sobolev spaces $\dot{W}^{1, p}\left(\mathbf{R}^{n}\right)$ are defined by demanding that all the first order distributional derivatives of $f$ lie in $L^{p}\left(\mathbf{R}^{n}\right)$.

Definition 2. Let $p>0$. We say that a tempered distribution $f$ on $\mathbf{R}^{n}$ belongs to the homogeneous (Hardy-)Sobolev space $\dot{H}^{1, p}\left(\mathbf{R}^{n}\right)$ if and only if $D_{j} f \in H^{p}\left(\mathbf{R}^{n}\right)$ for each $j=1, \ldots, n$. Moreover

$$
\|f\|_{\dot{H}^{1, p}\left(\mathbf{R}^{n}\right)}:=\sum_{j=1}^{n}\left\|D_{j} f\right\|_{H^{p}\left(\mathbf{R}^{n}\right)},
$$

whence we obtain a (quasi)Banach space modulo constants.

The notation used above follows Triebel's [30] convention and it deliberately avoids confusion for $p=1$, since obviously one has $\dot{H}^{1, p}\left(\mathbf{R}^{n}\right)=\dot{W}^{1, p}\left(\mathbf{R}^{n}\right)$ if $p>1$, while for $p=1$ this breaks down.

In the present paper our main interest lies in the case where the elements in $\dot{H}^{1, p}$ are honest locally integrable functions. This actually happens for $p \geq n /(n+1)$. The following result is well-known, but we obtain it as a corollary of our proof of Theorem 1, see Remark 8 below.

Proposition 3. Assume that $n /(n+1) \leq p \leq 1$ and let $p^{*}:=\frac{p n}{n-p}$ be the Sobolev conjugate exponent, so that $p^{*} \geq 1$. Then $\dot{H}^{1, p}\left(\mathbf{R}^{n}\right) \subset L_{l o c}^{p^{*}}\left(\mathbf{R}^{n}\right)$. Especially, the elements of $\dot{H}^{1, p}\left(\mathbf{R}^{n}\right)$ are locally integrable.

We next recall the definition of the spaces $M^{1, p}\left(\mathbf{R}^{n}\right)$ and their homogeneous counterparts $\dot{M}^{1, p}\left(\mathbf{R}^{n}\right)$.

Definition 4. Let $p>0$ and let $\Omega \subset \mathbf{R}^{n}$ be a subdomain. A measurable function $u$ belongs to the homogeneous Sobolev space $\dot{M}^{1, p}(\Omega)$ (defined in the sense of Hajłasz) if there is a function $g \in L^{p}(\Omega)$ and a set $E \subset \Omega$ of measure zero such that for all $x, y \in \Omega \backslash E$ we have the estimate

$$
|u(x)-u(y)| \leq|x-y|(g(x)+g(y)) .
$$

The corresponding quasi-norm is obtained by setting

$$
\|f\|_{\dot{M}^{1, p}(\Omega)}:=\inf \|g\|_{L^{p}(\Omega)},
$$

where the infimum is taken over all admissible functions $g$ in (4). The non-homogeneous space $M^{1, p}(\Omega)$ is obtained by requiring, in addition, that $f \in L^{p}(\Omega)$, and the norm for this space is defined by $\|f\|_{M^{1, p}(\Omega)}:=\|f\|_{\dot{M}^{1, p}(\Omega)}+\|f\|_{L^{p}(\Omega)}$.

Usually, we do not specifically mention the exceptional set $E$ since one may naturally allow the function $g$ to have value $\infty$ in a set of measure zero. If (4) holds for a certain measurable $g$ we say that $g \in D(u)$.

It is well-known that for $p>1$ one has $M^{1, p}\left(\mathbf{R}^{n}\right)=W^{1, p}\left(\mathbf{R}^{n}\right)$. Actually, in this case (see [13, Thm 2.2 and formula (2.5)]) there is a constant $c>0$ so that

$$
g:=c M(|D f|) \in D(u) .
$$


We note also that Hajłasz [14] characterized the space $\dot{W}^{1,1}\left(\mathbf{R}^{n}\right)$ by a condition related to $(4)$.

When $p<1$ the spaces $M^{1, p}(\Omega)$ are, of course, quasi-Banach spaces (modulo constant functions), and for $p \geq 1$ Banach spaces. For these and other basic facts on $M^{1, p}\left(\mathbf{R}^{n}\right)$ we refer to [13]. Especially, Hajłasz established an important extension [13, Thm. 8.7] of the Sobolev embedding theorem to the quasi-Banach case, which holds true for metric spaces satisfying a lower bound for the growth of the measure of balls. In the case of $\mathbf{R}^{n}$ a special case of Hajłasz's theorem states the following.

Proposition 5. Let $B \subset \mathbf{R}^{n}$ be a ball with radius $r$. Then, for any $u \in \dot{M}^{1, p}(2 B)$ with $0<p<n$, and $g \in D(u)$ one has the estimate

$$
\inf _{c \in \mathbf{R}}\left(f_{B}|u-c|^{p^{*}} d m\right)^{1 / p^{*}} \leq C r\left(f_{2 B} g^{p} d m\right)^{1 / p} .
$$

Here $p^{*}=\frac{n p}{n-p}$ and $C$ depends only on $p$ and $n$.

Above one may replace the domain of integration $2 B$ by any ball $\lambda B$, where $\lambda \in(1,2]$. If $p^{*} \geq 1$, or equivalently $p \geq n /(n+1)$, we see that an element $f \in M^{1, p}\left(\mathbf{R}^{n}\right)$ is locally integrable, whence it defines a distribution. A standard argument which uses Proposition 5 to compare mean values of $f$ in balls $B_{j}$ and $B_{j+1}$ with $B_{j}=$ $B\left(0,2^{j}\right) \subset \mathbf{R}^{n}$ shows that $u(x)(1+|x|)^{-m} \in L^{1}\left(\mathbf{R}^{n}\right)$ for large enough $m$. Hence $u$ (and, consequently, its derivatives) lie in $\mathcal{S}^{\prime}\left(\mathbf{R}^{n}\right)$.

We will apply the previous Proposition in our proof of Theorem 1. The other essential ingredient is Theorem 7 below, which provides the appropriate generalization of (5) to the case $p \leq 1$. In the proof of Theorem 7 we need the following well-known lemma (see e.g. [1, Lemma 3.29]), that is related to de Rham cohomology. We include a proof for completeness.

Lemma 6. Let $Q \subset \mathbf{R}^{n}$ be a cube and $\varphi \in C_{0}^{\infty}(Q)$. Then there are elements $\psi_{k} \in$ $C_{0}^{\infty}(Q), k=1, \ldots, n$ such that

$$
\varphi=\sum_{k=1}^{n} D_{k} \psi_{k}
$$

if and only if the condition $\int_{\mathbf{R}^{n}} \varphi d x=0$ is satisfied.

Proof. The stated condition is trivially necessary. In order to prove the sufficiency we apply induction on $n$. The case $n=1$ is evident, so suppose that the result holds true for a fixed $n \geq 1$ and let $Q=Q^{\prime} \times I \subset \mathbf{R}^{n+1}$, where $Q^{\prime} \subset \mathbf{R}^{n}$ is a cube and $I \subset \mathbf{R}$ is an interval. For $x \in \mathbf{R}^{n+1}$ we write $x=\left(x^{\prime}, x_{n+1}\right)$ where $x^{\prime}=\left(x_{1}, \ldots, x_{n}\right) \in \mathbf{R}^{n}$. Assume that $\varphi \in C_{0}^{\infty}(Q)$ has zero mean. Define

$$
h\left(x^{\prime}\right):=\int_{-\infty}^{\infty} \varphi\left(x^{\prime}, u\right) d u .
$$

Then $h \in C_{0}^{\infty}\left(Q^{\prime}\right)$ and, moreover, $h$ has zero mean. The induction hypothesis enables us to write

$$
h\left(x^{\prime}\right)=D_{1} h_{1}\left(x^{\prime}\right)+D_{2} h_{2}\left(x^{\prime}\right)+\ldots+D_{n} h_{n}\left(x^{\prime}\right)
$$

with $h_{j} \in C_{0}^{\infty}\left(Q^{\prime}\right)$ for $j=1, \ldots, n$. Finally, pick $a \in C_{0}^{\infty}(I)$ with $\int_{I} a(u) d u=1$ and observe that the desired decomposition is obtained by choosing

$$
\psi_{n+1}(x):=\int_{-\infty}^{x_{n+1}}\left(\varphi\left(x^{\prime}, u\right)-a(u) h\left(x^{\prime}\right)\right) d u
$$


and $\psi_{j}(x)=a\left(x_{n+1}\right) h_{j}\left(x^{\prime}\right)$ for $j=1, \ldots, n$.

If $x, y \in \mathbf{R}^{n}$ we denote by $B_{x, y}$ the ball with the segment between $x$ and $y$ as a diameter. Our next result is of independent interest. Observe that the only assumption on $f$ below is the local integrability. The maximal functions appering on the right hand side of (6) are well defined for any distribution, and for a suitably chosen $L^{1}$-function $f$ they could well take the value $\infty$ at every point.

Theorem 7. For any $N \geq 1$ there exists a constant $c=c(N, n)$ with the following property: If $B \in \mathbf{R}^{n}$ is a ball and $f \in L^{1}(2 B)$, then there is a set $E \subset B$ of measure zero such that for every $x, y \in B \backslash E$ it holds that

$$
|f(x)-f(y)| \leq c|x-y|\left(\mathcal{M}_{|x-y|, N} D f(x)+\mathcal{M}_{|x-y|, N} D f(y)\right) .
$$

Proof. Fix $N \geq 1$. By rotational symmetry we may assume that $x-y=r e_{1}$, where $e_{1}$ is the first unit coordinate vector and $r=|x-y|>0$. Let $\varphi \in C_{0}^{\infty}(B(0,1))$ be a fixed test function with $\int_{\mathbf{R}^{n}} \varphi d x=1$. Choose $k_{0} \geq 1$ so that $2^{k_{0}-1} \geq \sqrt{n}$.

Let us denote

$$
A_{k}=\int_{\mathbf{R}^{n}} f(z+x) 2^{n\left(k+k_{0}\right)} r^{-n} \varphi\left(2^{k_{0}+k} r^{-1} z\right) d z \quad \text { for } k \geq 0 .
$$

and analogously

$$
B_{k}=\int_{\mathbf{R}^{n}} f(z+y) 2^{n\left(k+k_{0}\right)} r^{-n} \varphi\left(2^{k_{0}+k} r^{-1} z\right) d z \quad \text { for } k \geq 0 .
$$

We apply Lemma 6 to the function $\varphi(\cdot)-2^{n} \varphi(2 \cdot)$ and write

$$
\varphi(z)-2^{n} \varphi(2 z)=\sum_{j=1}^{n} D_{k} \psi_{j}(z) \quad \text { for } z \in \mathbf{R}^{n}
$$

with $\psi_{j} \in C_{0}^{\infty}\left([-1,1]^{n}\right)$ for $1 \leq j \leq n$. An integration by parts yields that

$$
\begin{aligned}
\left|A_{k}-A_{k+1}\right| & =\left|\sum_{j=1}^{n} \int_{\mathbf{R}^{n}} f(z+x) 2^{n\left(k+k_{0}\right)} r^{-n} D_{j} \psi_{j}\left(2^{k_{0}+k} r^{-1} z\right) d z\right| \\
& =2^{-k_{0}-k} r\left|\sum_{j=1}^{n}\left\langle D_{j} f(\cdot+x), 2^{n\left(k+k_{0}\right)} r^{-n} \psi_{k}\left(2^{k_{0}+k} r^{-1} \cdot\right)\right\rangle\right| \\
& \leq C(n, N) r 2^{-k} \mathcal{M}_{|x-y|, N} D f(x) \quad \text { for } k \geq 0 .
\end{aligned}
$$

Similarly

$$
\left|B_{k}-B_{k+1}\right| \leq C(n, N) r 2^{-k} \mathcal{M}_{|x-y|, N} D f(y) \text { for } k \geq 0 .
$$

It remains to estimate the difference $\left|A_{0}-B_{0}\right|$. Denote

$$
\widetilde{\varphi}(z)=2^{n k_{0}}\left[\varphi\left(2^{k_{0}} z\right)-\varphi\left(2^{k_{0}}\left(z+e_{1}\right)\right)\right] .
$$

Then $\int_{\mathbf{R}^{n}} \widetilde{\varphi} d x=0$. By the choice of $k_{0}$ we may apply Lemma 6 to $\widetilde{\varphi}$ to obtain functions $\widetilde{\psi}_{k} \in C_{0}^{\infty}\left(B(0,1) \cup B\left(e_{1}, 1\right)\right)$ such that $\widetilde{\varphi}=\sum_{k=1}^{n} D_{k} \widetilde{\psi}_{k}$. With the help of suitable cut-off functions we may for each $k$ decompose $k \widetilde{\psi}_{k}=\widetilde{\psi}_{k, 1}+\widetilde{\psi}_{k, 2}$, where $\operatorname{supp}\left(\widetilde{\psi}_{k, 1}\right) \subset B(0,1)$ and $\operatorname{supp}\left(\widetilde{\psi}_{k, 2}\right) \subset B\left(e_{1}, 1\right)$. Then by translating, scaling and integrating by parts we obtain as before that

$$
\left|A_{0}-B_{0}\right| \leq \operatorname{Cr}\left(\mathcal{M}_{|x-y|, N} D f(x)+\mathcal{M}_{|x-y|, N} D f(y)\right) .
$$


Finally, observe that if both $x$ and $y$ are Lebesgue points of $f$ we have $f(x)=$ $\lim _{k \rightarrow \infty} A_{k}$ and $f(y)=\lim _{k \rightarrow \infty} B_{k}$. The estimates (7)-(9) thus yield that

$$
\begin{aligned}
|f(x)-f(y)| & \leq\left|A_{0}-B_{0}\right|+\sum_{k=0}^{\infty}\left(\left|A_{k}-A_{k+1}\right|+\left|B_{k}-B_{k+1}\right|\right. \\
& \leq C^{\prime} r\left(\mathcal{M}_{|x-y|, N} D f(y)+\mathcal{M}_{|x-y|, N} D f(y)\right),
\end{aligned}
$$

and this finishes the proof of the Theorem.

We are now ready for the proof of our main theorem.

Proof of Theorem 1. Assume first that $n /(n+1)<p \leq 1$ and $f$ is a Schwartz distribution on $\mathbf{R}^{n}$ such that $D_{j} f \in H^{p}\left(\mathbf{R}^{n}\right)$ for all $1 \leq j \leq n$. By Proposition 3 we have that $f \in L_{l o c}^{1}\left(\mathbf{R}^{n}\right)$ (see also Remark 8 below). Hence Theorem 7 applies and we deduce that outside an exeptional set of measure zero $f$ satisfies the inequality (4) with the choice $g=\mathcal{M}_{1} D f$. By the assumptions we have $g \in L^{p}\left(\mathbf{R}^{n}\right)$. Moreover, there is the estimate $\|g\|_{L^{p}} \leq C \sum_{j=1}^{n}\left\|D_{j} f\right\|_{L^{p}}$. We have shown that $f \in \dot{M}^{1, p}\left(\mathbf{R}^{n}\right)$ with the correct bound for the (quasi)norm.

For the converse, assume next that $f \in \dot{M}^{1, p}\left(\mathbf{R}^{n}\right)$ and $p>n /(n+1)$. Fix $j \in$ $\{1, \ldots, n\}$. We are to show that $D_{j} f \in H^{p}\left(\mathbf{R}^{n}\right)$. For that end, we denote by $B(x, r)$ the ball of radius $r$ and center $x$. Recall also that the support of $\psi$ is contained in the open unit ball, and denote $C_{0}:=\|D \psi\|_{\infty}$. Since obviously $f_{\mid B(x, 2 r)} \in \dot{M}^{1, n /(n+1)}(B(x, 2 r))$, we may apply Proposition 5 with $p=n /(n+1)$ and obtain that

$$
f_{B(x, r)}\left|f-f_{B(x, r)}\right| d m \leq C_{0} r\left(f_{B(x, 2 r)} g^{n /(n+1)} d m\right)^{(n+1) / n},
$$

where $f_{B(x, r)}$ stands for the mean value of $f$ in the ball $B(x, 2 r)$. Apply the above inequality to compute

$$
\begin{aligned}
\mathcal{M}_{\psi}\left(D_{j} u\right)(x) & =\sup _{t>0}\left|\left\langle D_{j} f, t^{-n} \psi((x-\cdot) / t)\right\rangle\right| \\
& =\sup _{t>0}\left|\left\langle f, t^{-n-1}\left(D_{j} \psi\right)((x-\cdot) / t)\right\rangle\right| \\
& =\sup _{t>0}\left|t^{-n-1} \int_{B(x, t)}\left(f(y)-f_{B(x, t)}\right)\left(D_{j} \psi\right)((x-y) / t) d y\right| \\
& \leq C_{0} \sup _{t>0} t^{-1} f_{B(x, t)}\left|f(y)-f_{B(x, t)}\right| d y \\
& \leq 2 C C_{0} \sup _{t>0}\left(f_{B(x, 2 t)} g^{n /(n+1)}\right)^{(n+1) / n} \\
& \leq C^{\prime}\left(M\left(g^{n /(n+1)}\right)(x)\right)^{(n+1) / n} .
\end{aligned}
$$

By the Hardy-Littlewood theorem and the assumption $g \in L^{p}\left(\mathbf{R}^{n}\right)$ we deduce that $M\left(g^{n /(n+1)}\right) \in L^{q}\left(\mathbf{R}^{n}\right)$ where $q=(n+1) p / n$. This shows that $\mathcal{M}_{\psi}\left(D_{i} u\right) \in L^{p}\left(\mathbf{R}^{n}\right)$, as was to be shown.

Remark 8. Actually we may easily bypass the use of Proposition 3 in the above proof. Namely, consider a Schwartz distribution $f$ such that such that $D_{j} f \in H^{p}\left(\mathbf{R}^{n}\right)$ for all $1 \leq j \leq n$. Choose smooth convolution approximations $f_{k}$ of $f$ so that $\| D_{j} f_{k}-$ 
$D_{j} f \|_{H^{p}\left(\mathbf{R}^{n}\right)} \leq 2^{-k}$ for each $k \geq 1$ and $j=1, \ldots, n$. By applying the above proof on each difference $f_{k+1}-f_{k}$ and by writing $f=f_{1}+\sum_{k=1}^{\infty}\left(f_{k+1}-f_{k}\right)$ we obtain the desired result for $f$. In turn, Proposition 3 is now obtained as a consequence of Theorem 1 and Proposition 5.

Note that according to Proposition 3 we could as well assume a priori that $f$ is a tempered distribution in the following definition.

Definition 9. Let $p<n /(n+1)$. We say that a locally integrable $f$ on $\mathbf{R}^{n}$ belongs to the (non-homogeneous) Hardy-Sobolev space $H^{1, p}\left(\mathbf{R}^{n}\right)$ if $D_{j} f \in H^{p}\left(\mathbf{R}^{n}\right)$ for each $j=1, \ldots, n$ and $f \in L^{p}\left(\mathbf{R}^{n}\right)$. Moreover, we set

$$
\|f\|_{H^{1, p}\left(\mathbf{R}^{n}\right)}:=\sum_{j=1}^{n}\left\|D_{j} f\right\|_{H^{p}\left(\mathbf{R}^{n}\right)}+\|f\|_{L^{p}\left(\mathbf{R}^{n}\right)} .
$$

The following immediate corollary of Theorem 1 verifies (2).

Corollary 10. Let $p>n /(n+1)$. Then $M^{1, p}\left(\mathbf{R}^{n}\right)=H^{1, p}\left(\mathbf{R}^{n}\right)$ with equivalent norms.

It is well-known that bounded sets in the Hardy-spaces are weakly compact (see [27, p. 127]) in the sense that any bounded sequence contains a subsequence that converges in the sense of distributions to an element in the same space. We thus obtain the following compactness result for the spaces $M^{1, p}\left(\mathbf{R}^{n}\right)$.

Corollary 11. Let $p>n /(n+1)$. Bounded sets in the space $M^{1, p}\left(\mathbf{R}^{n}\right)$ are weakly compact in the following sense: if $\left(f_{k}\right)$ is a norm-bounded sequence in $M^{1, p}\left(\mathbf{R}^{n}\right)$, then there is an element $f \in M^{1, p}\left(\mathbf{R}^{n}\right)$ and a subsequence $f_{k_{\ell}}$ so that

$$
f_{k_{\ell}} \rightarrow f \quad \text { in } L_{l o c}^{1} \quad \text { as } \ell \rightarrow \infty \text {. }
$$

Proof. Consider a norm-bounded sequence $\left(f_{k}\right)$ in $M^{1, p}\left(\mathbf{R}^{n}\right)$. By the above mentioned weak compactness of the Hardy spaces we may pass to a subsequence and assume that for each $j$ it holds that $D_{j} f_{k} \rightarrow h_{j}$ in the sense of distributions. Obviously $h_{j}=D_{j} g$ for some distribution $g$. Then $g \in H^{1, p}\left(\mathbf{R}^{n}\right)$ and the rest follows by an application of Corollary 10 via the known compactness [30] of the embedding (restriction map) $H^{1, p}\left(\mathbf{R}^{n}\right) \subset L^{1}(B)$ for any ball $B \subset \mathbf{R}^{n}$.

Regarding applications to $H^{1, p}$ spaces we point out that the truncation stability (or, more generally, Lipschitz stability) of these spaces, proven by Janson [18], is an immediate consequence of our Theorem 1.

Remark 12. One may check that $H^{1, p}\left(\mathbf{R}^{n}\right)=\dot{F}_{p, 2}^{1}\left(\mathbf{R}^{n}\right) \cap L^{p}\left(\mathbf{R}^{n}\right)$, where $\dot{F}_{p, 2}^{1}\left(\mathbf{R}^{n}\right)$ is the homogeneous Triebel space, see [30, Section 5].

Remark 13. In the proof of Theorem 1 it would be possible to partially apply the existing results on Hardy-Sobolev spaces [9], [18], [24], [25]. However, our proof is direct and simple and it employs just the maximal function definition of the Hardy spaces. It is perhaps of interest to also note that one may also prove first half of Theorem 
1, i.e. the inclusion $\dot{H}^{1, p}\left(\mathbf{R}^{n}\right) \subset \dot{M}^{1, p}\left(\mathbf{R}^{n}\right)$ by applying the atomic decomposition of the Hardy spaces (see [27, III.2]). This is done with the aid of the representation

$$
f=\sum_{j=1}^{n} I_{1} R_{j} D_{j} f+\text { const }
$$

where the $R_{j}$ :s are the Riesz transforms and the Riesz potential $I_{1}$ corresponds to the Fourier-multiplier $\widehat{f} \mapsto|\xi|^{-1} \widehat{f}$. Since the Riesz transforms are bounded on the Hardy spaces, and we are dealing with the case $p \leq 1$, it turns out that it is enough to show that for each $H^{p}$-atom $a$ we have $I_{1} a \in \dot{M}^{1, p}\left(\mathbf{R}^{n}\right)$. Moreover, by scaling and translation invariance one may assume that the atom $a$ is related to the unit ball $B(0,1)$, whence one may prove by hand that $I_{1} a \in \dot{M}^{1, p}\left(\mathbf{R}^{n}\right)$. On the other hand, Theorem 7 is a more convenient tool since it bybasses the atomic theory and, more importantly, it is a local result which applies directly to spaces defined on subdomains of $\mathbf{R}^{n}$, see Section 3 below.

Remark 14. It is natural to also consider exponents $p \leq n /(n+1)$ and ask for the right analogue for our characterization of the Hardy-Sobolev spaces. Moreover, we do not know if Theorem 1 holds true as such in the case $p=n /(n+1)$.

\section{Local spaces and spaces on subdomains of $\mathbf{R}^{n}$}

The elements of $H^{p}\left(\mathbf{R}^{n}\right)$-spaces satisfy moment conditions, e.g. any function from $H^{1}\left(\mathbf{R}^{n}\right)$ has mean zero. In order to relax this condition and to obtain localizable spaces, one defines ([10], see also $\left[27\right.$, Ch. 3, Sec. 5.17]) the local Hardy space $h^{p}\left(\mathbf{R}^{n}\right)$ by restricting, in the definition of the maximal operator $\mathcal{M}_{\psi}$, the range of $t$ to the interval $(0, \rho]$, where $\rho>0$ is a fixed positive number. Define

$$
\mathcal{M}_{\psi, \rho} f(x):=\sup _{0<t \leq \rho}\left|f * \psi_{t}(x)\right|,
$$

whence a tempered distribution is said to belong to $h^{p}\left(\mathbf{R}^{n}\right)(p>0)$ if and only if $\mathcal{M}_{\psi, \rho} f \in L^{p}\left(\mathbf{R}^{n}\right)$. One sets $\|f\|_{h^{p}}:=\left\|\mathcal{M}_{\psi, \rho} f\right\|_{L^{p}}$. The above definition does not depend on $\rho>0$ or on $\psi$. In particular, different values of $\rho$ lead to equivalent (quasi)norms. Finally, in terms of grand maximal functions

$$
f \in h^{p}\left(\mathbf{R}^{n}\right) \quad \text { if and only if } \quad \mathcal{M}_{\rho, N} f \in L^{p}\left(\mathbf{R}^{n}\right) .
$$

as soon as $N \geq N_{0}(p, n)$.

The local Hardy spaces form a more flexible class than the Hardy spaces. There are no more global moment conditions, the elements in $h^{p}$ can be localized and they are (at least locally) invariant under diffeomorphisms.

The local Hardy-Sobolev space is obtained in a natural way:

Definition 15. Let $p>0$. We say that a tempered distribution $f$ on $\mathbf{R}^{n}$ belongs to the homogeneous Hardy-Sobolev space $\dot{h}^{1, p}\left(\mathbf{R}^{n}\right)$ if and only if $D_{j} f \in h^{p}\left(\mathbf{R}^{n}\right)$ for each $j=1, \ldots, n$. Moreover

$$
\|f\|_{h^{1, p}\left(\mathbf{R}^{n}\right)}:=\sum_{j=1}^{n}\left\|D_{j} f\right\|_{h^{p}\left(\mathbf{R}^{n}\right)} .
$$

In case $p<1$ the above space is a quasi-Banach space modulo constants. Similarly, in case $p \geq n /(n+1)$ we say that $f \in h^{1, p}\left(\mathbf{R}^{n}\right)$ if $D_{j} f \in h^{p}\left(\mathbf{R}^{n}\right)$ for each $j=1, \ldots, n$, and $f \in L^{p}\left(\mathbf{R}^{n}\right)$. 
The last sentence above makes sense since locally the elements of $h^{p}$ coincide with elements in $H^{p}$ whence Proposition 3 easily implies $\dot{h}^{1, p}\left(\mathbf{R}^{n}\right) \subset L_{l o c}^{1}\left(\mathbf{R}^{n}\right)$.

We now verify a counterpart of Theorem 1 for the local spaces. Below one may replace the condition $|x-y| \leq 1$ by $|x-y| \leq c$, where $c>0$ is an arbitrary constant.

Theorem 16. Let $n \geq 1$ and $\frac{n}{n+1}<p \leq 1$. Then a distribution $f \in S^{\prime}\left(\mathbf{R}^{n}\right)$ belongs to $\dot{h}^{1, p}\left(\mathbf{R}^{n}\right)$ if and only if $f$ is locally integrable and there is a function $g \in L^{p}$ such that

$$
|f(x)-f(y)| \leq|x-y|(g(x)+g(y)), \quad \text { for }|x-y| \leq 1 \text { and } x, y \in \mathbf{R}^{n} \backslash E
$$

where $E$ is a set of measure zero. Moreover, one has the equivalence of (quasi)norms

$$
\|f\|_{h^{1, p}} \sim \inf \|g\|_{p}
$$

where the infimum is taken over all admissible functions $g$ in (11), and one identifies functions differing only by a constant.

Proof. Let us first assume that condition (11) holds true. If we replace $\sup _{t>0}$ by $\sup _{t \leq 1 / 4}$ in the second part of the proof of Theorem 1 we may again apply Proposition 5 in a similar manner to deduce that $\mathcal{M}_{\psi, 1 / 4}\left(D_{k} f\right)(x) \in L^{p}\left(\mathbf{R}^{n}\right)$. It follows that $f \in \dot{h}^{1, p}\left(\mathbf{R}^{n}\right)$.

In order to prove the converse, observe that according to Theorem 7 condition (11) is satisfied with the choice $g:=c \max _{1 \leq j \leq n} \mathcal{M}_{N, 1} D_{j} f$. The assumption $D_{j} f \in h^{p}$ now implies that $g \in L^{p}$ as soon as $N$ is large enough. Here, as also in the first part of the proof, the corresponding quantitative statement is obvious.

We now turn to the study of Hardy-Sobolev spaces on subdomains of the Euclidean space. A natural and simple definition of Hardy spaces that works for all subdomains $\Omega \subset \mathbf{R}^{n}$ was given by Miyachi [22]. We recall a definition that is directly equivalent with Miyachi's definition. Thus, let $\Omega \subset \mathbf{R}^{n}$ be a domain, $p \in(0,1)$ and $N>$ $\max \left(0, n\left(\frac{1}{p}-1\right)\right)$. If $f \in \mathcal{D}^{\prime}(\Omega)$ one defines

$$
f \in H^{p}(\Omega) \quad \text { if and only if }\|f\|_{H^{p}(\Omega)}=:\left\|\mathcal{M}_{d(x, \partial \Omega) / 2, N} f\right\|_{p}<\infty
$$

It follows from Miyachi's results that the above is equivalent with the original definition [22]. Similarly, we see immediately that one may replace above $\mathcal{M}_{d(x, \partial \Omega) / 2} f$ by $\mathcal{M}_{a d(x, \partial \omega)} f$ for any $a \in(0,1)$, or by a the more standard maximal function $\sup _{0<t \leq d(x, \partial \Omega)}\left|f * \psi_{t}\right|$, where the fixed test function $\psi \in C_{0}^{\infty}(B(0,1))$ has nonzero mean. All the (quasi)norms so obtained are mutually equivalent. Let us also note that prior to [22], Jonsson, Sjögren, and Wallin [19] defined Hardy spaces on fairly general subsets of $\mathbf{R}^{n}$ in terms of suitable atoms.

Recall that the spaces $\dot{M}^{1, p}(\Omega)$ and $M^{1, p}(\Omega)$ were defined already in the previous section. The spaces $\dot{M}_{\text {ball }}^{1, p}(\Omega)$ and $M_{\text {ball }}^{1, p}(\Omega)$ are defined exactly in the same manner but for one difference: in the definition of these spaces the condition (4) is assumed to hold only for points $x, y \in \Omega \backslash F$ that satisfy the condition

$$
|x-y| \leq \frac{1}{4} \min (d(x, \partial \Omega), d(y, \partial \Omega)) .
$$

After the proof of Theorem 19 below it is clear that one may replace in the last condition above the constant $1 / 4$ by any constant strictly less than one.

The Hardy-Sobolev spaces on $\Omega$ are defined in the obvious manner. 
Definition 17. Let $\Omega \subset \mathbf{R}^{n}$ be a domain and $p>0$. A measurable function $u: \Omega \rightarrow \mathbf{R}$ belongs to the homogeneous Hardy-Sobolev space $\dot{H}^{1, p}(\Omega)$ if $D_{j} f \in H^{p}(\Omega)$ for all $j=1, \ldots, n$. The related seminorm is obtained by setting $\|f\|_{\dot{H}^{1, p}(\Omega)}:=$ $\sum_{j=1}^{n}\left\|D_{j} f\right\|_{H^{p}(\Omega)}$. In case $p \geq n /(n+1)$ we say that $f \in H^{1, p}(\Omega)$ if additionally $f \in L^{p}(\Omega)$, and the corresponding (quasi)norm is defined in the obvious manner.

Theorem 18. Let $n \geq 1$ and $\frac{n}{n+1}<p \leq 1$, and assume that $\Omega \subset \mathbf{R}^{n}$ is a domain. Then

$$
H^{1, p}(\Omega)=M_{\text {ball }}^{1, p}(\Omega) \quad \text { and } \quad \dot{H}^{1, p}(\Omega)=\dot{M}_{\text {ball }}^{1, p}(\Omega),
$$

with equivalence of the (quasi)norms.

Proof. It is enough to prove the latter equality. Assume first that $f \in H^{1, p}(\Omega)$. Let $|x-y| \leq \frac{1}{4} \min (d(x, \partial \Omega), d(y, \partial \Omega))$. Since the first derivatives of $f$ are locally in $h^{1, p}$, it easily follows that $f \in L_{l o c}^{1}$. We may apply Theorem 7 in the ball $B\left(\frac{1}{2}(x+\right.$ $\left.y), \frac{1}{3} d(x, \partial \Omega)\right)$ in order to obtain the inequality (4) with the choice

$$
g(x)=\mathcal{M}_{\frac{1}{2} d(x, \partial \Omega), N} D f(x) .
$$

By definition it holds that $g \in L^{p}(\Omega)$.

The converse follows exactly as the proof of the second part of Theorem 1 . One just uses the observation that $\sup _{\varphi \in C_{0, N}^{\infty}(B(x, r))}\left\|D_{k} \varphi\right\|_{1} \leq c(n, N) r^{-1}$ for each $k=$ $1, \ldots, n$.

For simplicity, from now on we state our results only for non-homogeneus spaces. The reader will have no difficulty in formulating the corresponding results for the homogeneous spaces. After our previous results it is of interest to find conditions on $\Omega$ that would quarantee that the obvious inclusion $M^{1, p}(\Omega) \subset M_{\text {ball }}^{1, p}(\Omega)$ becomes equality. Let us recall for that end the definition of uniform domains. One says that a domain $\Omega \subset \mathbf{R}^{n}$ is uniform if there is a constant $c>0$ such that for all $x, y \in \Omega$ there is a rectifiable path $\gamma:[0, T] \rightarrow \Omega$, parametrized by arclength, with $\gamma(0)=x$, $\gamma(T)=y$, and such that $T \leq c|x-y|$ together with

$$
B\left(\gamma(t), \frac{1}{c} \min (t, T-t)\right) \subset \Omega \text { for } t \in(0, T) .
$$

Theorem 19. Let $n \geq 1$ and $\frac{n}{n+1}<p \leq 1$, and assume that $\Omega \subset \mathbf{R}^{n}$ is a uniform domain. Then $M_{\text {ball }}^{1, p}(\Omega)=M^{1, p}(\Omega)$ and, especially

$$
H^{1, p}(\Omega)=M^{1, p}(\Omega)
$$

with equivalent (quasi)norms.

Proof. Choose arbitrary $x, y \in \Omega$. From the uniformity condition we deduce easily that there is a chain of balls $B_{k}$ resembling a cigar that joins the points $x$ and $y$. To be more specific, there are balls $B_{k}:=B\left(z_{k}, r_{k}\right)$ with $k \in \mathbf{Z}$ and $z_{k} \in \Omega$ such that for each $k$ one has $6 B_{k} \subset \Omega, r_{k} \geq \frac{1}{c^{\prime}} \min \left(d\left(z_{k}, x\right), d\left(z_{k}, y\right)\right), B_{k} \cap B_{k+1} \neq \emptyset$, and $r_{k} / 2 \leq r_{k+1} \leq 2 r_{k}$. In addition, $\lim _{k \rightarrow \infty} d\left(x, B_{k}\right)=0=\lim _{k \rightarrow-\infty} d\left(y, B_{k}\right)$. Finally, we may assume that $\sum_{k \in \mathbf{Z}} r_{k} \leq c^{\prime}|x-y|$ 
Let $f \in M_{\text {ball }}^{1, p}(\Omega)$. We compare in a standard manner the consequtive mean values $f_{B_{k}}$ by applying the Poincare inequality of Proposition 5 in the ball $3 B_{k}$

$$
\begin{aligned}
\left|f_{B_{k}}-f_{B_{k+1}}\right| & \lesssim f_{3 B_{k}}\left|f-f_{3 B_{k}}\right| d x \lesssim r_{k}\left(f_{6 B_{k}} g^{n /(n+1)} d m\right)^{(n+1) / n} \\
& \lesssim r_{k}\left(\left(M g^{n /(n+1)}(x)\right)^{(n+1) / n}+\left(M g^{n /(n+1)}(y)\right)^{(n+1) / n}\right) .
\end{aligned}
$$

Above we assumed $g$ to be continued as zero outside $\Omega$, and the last estimate followed by observing that one of the points $x, y$ is contained in $c^{\prime} B_{k}$.

If $x, y$ are Lebesgue points of $f$ we have $|f(x)-f(y)| \leq \sum_{k \in \mathbf{Z}}\left|f_{B_{k}}-f_{B_{k+1}}\right|$. By summing over $k$ in our previous estimate it follows that

$$
|f(x)-f(y)| \leq|x-y|(\widetilde{g}(x)+\widetilde{g}(y))
$$

where $\widetilde{g}=c^{\prime} C^{\prime}\left(\left(M g^{n /(n+1)}\right)^{(n+1) / n}\right)_{\mid \Omega}$. The conclusion follows from Theorem 18 since by the standard Hardy-Littlewood maximal inequality $\widetilde{g} \in L^{p}(\Omega)$.

We next consider extensions from bounded domains to the whole space $\mathbf{R}^{n}$. We do not aim for most general results here (see Remark 22 below), but show how our results can be used to give a transparent new proof of the following result of Miyachi [23].

Theorem 20. (Miyachi) Let $n \geq 1$ and $\frac{n}{n+1}<p \leq 1$, and assume that $\Omega \subset \mathbf{R}^{n}$ is a bounded uniform domain. Then there is a bounded linear extension operator from $H^{1, p}(\Omega)$ into $H^{1, p}\left(\mathbf{R}^{n}\right)$. A fortiori,

$$
H^{1, p}(\Omega)=\left\{f_{\mid \Omega}: f \in H^{1, p}\left(\mathbf{R}^{n}\right)\right\} .
$$

The above Theorem is a direct consequence of Theorems 16 and 19 as soon as we verify the following Proposition.

Proposition 21. Let $n \geq 1$ and $\frac{n}{n+1}<p \leq 1$, and assume that $\Omega \subset \mathbf{R}^{n}$ is a bounded uniform domain. Then there is a bounded linear extension operator from $M^{1, p}(\Omega)$ into $H^{1, p}\left(\mathbf{R}^{n}\right)$. A fortiori,

$$
H^{1, p}(\Omega)=\left\{f_{\mid \Omega}: f \in H^{1, p}\left(\mathbf{R}^{n}\right)\right\} .
$$

Proof. Assume that $f \in M^{1, p}(\Omega)$ and $g \in L^{p}(\Omega)$ so that the condition (4) is satisfied. We assume that $g$ is continued as zero outside $\Omega$ and set $g_{1}:=\left(M g^{\widetilde{p}}\right)^{1 / \widetilde{p}}$, where as before $\widetilde{p}$ is chosen from the interval $(n /(n+1), p)$ so that $g_{1} \in L^{p}\left(\mathbf{R}^{n}\right)$. Assume that $\bar{B}(x, r), \bar{B}^{\prime}\left(x^{\prime}, r^{\prime}\right) \subset \Omega$ are two closed balls (or points). such that $2 r \leq$ $d(x, \partial \Omega)$ and $2 r^{\prime} \leq d\left(x^{\prime}, \partial \Omega\right)$. An easy modification of the proof of Theorem 19 yields the estimate:

$$
\left|f_{B}-f_{B^{\prime}}\right| \leq c\left(\left|x-x^{\prime}\right|+r+r^{\prime}\right)\left(\inf _{z \in B} g_{1}(z)+\inf _{z^{\prime} \in B^{\prime}} g_{1}\left(z^{\prime}\right)\right) .
$$

If, for example, $r=0$, we replace $f_{B}$ above by $f(x)$ and the corresponding infimum in the right hand side by $g_{1}(x)$. This makes sense as long as $x$ is a Lebesgue point of $f$.

For $\varepsilon>0$ set $A_{\varepsilon}:=\left\{x \in \mathbf{R}^{n} \backslash \Omega: d(x, \Omega) \leq \varepsilon\right\}$. We may choose $\varepsilon_{0}>0$ and a Whitney type cover of a neighbourhood of $\Omega$ by balls $\left\{B_{i}\right\}_{i=1}^{\infty}$ so that for the set $A:=\cup_{i=1}^{\infty} B_{i}$ it holds that $A_{4 \varepsilon_{0}} \subset A \subset A_{10 \varepsilon_{0}}$. Moreover, for each $i$ the balls $B_{i}=: B\left(y_{i}, \rho_{i}\right)$ satisfy $d\left(y_{i}, \Omega\right) \leq 4 \rho_{i} \leq 2 d\left(y_{i}, \Omega\right)$ and the inflated balls $2 B_{i}$ 
have bounded overlap. Finally, the situation can be arranged so that we may also pick a partition of unity that consist of functions $h_{i}$ such that $\operatorname{supp} h_{i} \subset B_{i}$, and $0 \leq h_{i} \leq 1$ together with $\left\|D h_{k}\right\|_{\infty} \leq c / \rho_{i}$. The desired partition of unity property may be expressed as follows: $\chi_{A_{2 \varepsilon_{0}}} \leq \sum_{i} h_{i} \leq \chi_{A_{10 \varepsilon_{0}}}$.

For each ball $B_{i}$ we may, by the uniformity of $\Omega$, select a ball $B_{i}^{\prime}:=B_{i}^{\prime}\left(y_{i}^{\prime}, r_{i}^{\prime}\right) \subset \Omega$ that satisfies uniformly $\operatorname{diam}\left(B_{i}\right) \sim \operatorname{diam}\left(B_{i}^{\prime}\right) \sim d\left(y_{i}^{\prime}, y_{i}\right) \sim d\left(B_{1}^{\prime}, \partial \Omega\right)$. The required extension $F$ of $f$ to the whole space $\mathbf{R}^{n}$ is then simply defined by choosing $F(x)=f(x)$ for $x \in \Omega$, and

$$
F(x)=\sum_{i=1}^{\infty}\left(f_{B_{i}} f d x\right) h_{i}(x) \text { for } x \in \mathbf{R}^{n} \backslash \bar{\Omega} .
$$

Observe that by the uniformity of $\Omega$ we may ignore $\partial \Omega$ since it is of measure zero.

We claim that condition (1) is satisfied for a.e. $x, y$ with $g_{2}$ in place of $g$, where $g_{2}:=\left(M g_{1}^{\widetilde{p}}\right)^{1 / \widetilde{p}} \in L^{p}\left(\mathbf{R}^{n}\right)$. The verification of this is a pretty routine using (13), and hence we just outline it. The details will possess no difficulties for the reader.

Consider arbitrary $x, y \in A_{\varepsilon_{0}} \cup \Omega$. We claim that condition (1) is satisfied for a.e. such points $x, y$. For $x, y \in \Omega$ this is evident, so assume that $x \in A_{2 \varepsilon_{0}} \backslash \bar{\Omega}$, say $x \in B_{i}=B\left(y_{i}, r_{i}\right)$. The argument will be divided into different cases depending on the relative location of the point $y$. Observe that $F(x)$ is a convex combination of averages of $f$ over balls $B_{i_{1}}^{\prime}, \ldots, B_{i_{\ell}}^{\prime} \subset \Omega$, say, whose distance to the boundary $\partial \Omega$ is comparable to their size and to their distance to $x$. Moreover, $\ell \leq C$ uniformly.

If $y \in \Omega$, it is thus enough to estimate differences of the form $\left|f(y)-f_{B_{i_{k}}^{\prime}}\right|$. We apply (13) and obtain immediately an estimate of the desired type, since in the present situation $g_{2}(x)$ dominates the quantity $\inf _{z \in B_{i_{k}}^{\prime}} g_{1}(z)$, and $|x-y| \gtrsim d\left(x, \cup_{k=1}^{\ell} f_{B_{i_{k}}^{\prime}}\right)$.

Let then also $y \in A_{2 \varepsilon_{0}} \backslash \bar{\Omega}$. whence the value $F(y)$ is likewise computed as a convex combination over averages in certain balls of the cover. We consider first the case where $|x-y| \geq c^{\prime} \max (d(x, \partial \Omega), d(y, \partial \Omega))$. If $B_{j}^{\prime}=B\left(y_{j}^{\prime}, r_{j}^{\prime}\right)$ is one of these balls, it is enough to estimate the difference $\left|f_{B_{i}^{\prime}}-f_{B_{j}^{\prime}}\right|$. This is done essentially as in the previous paragraph again by applying (13).

The remaining possibility is that $|x-y| \leq c^{\prime} \max (d(x, \partial \Omega), d(y, \partial \Omega))$. If $c^{\prime}$ is chosen appropriately, the properties of the Whitney type cover imply that distance of both $x$ and $y$ from the boundary $\partial \Omega$ are comparable to $r:=d(x, \partial \Omega)$ and the radii of the balls $B_{k}$ that contain one of the points $x, y$ are comparable to $r$, and the distance between these balls is less or comparable to $r$. Assume that $\rho_{k_{1}}, \ldots, \rho_{k_{m}}$ are the functions of the partition of unity that are nonzero at $x$ or at $y$. Denote $a_{u}:=f_{B_{k_{u}}^{\prime}} f_{k}$. The estimate (13) can be used to check that

$$
B:=\max _{1 \leq u, u^{\prime} \leq m}\left|a_{u}-a_{u}^{\prime}\right| \leq c^{\prime \prime} r\left(g_{2}(x)+g_{2}(y)\right) .
$$

Hence, as the $h_{i}$ :s form a partition of unity, we may estimate

$$
|F(x)-F(y)| \leq B \sum_{u=1}^{m}\left|h_{k_{u}}(x)-h_{k_{u}}(y)\right| .
$$

The desired upper bound for $|F(x)-F(y)|$ now follows by combining the previous estimates with the bound $\left|h_{k_{u}}(x)-h_{k_{u}}(y)\right| \leq|x-y|\left\|D h_{k_{u}}\right\|_{\infty} \leq C|x-y| / r$.

We have shown that $F \in \dot{M}^{1, p}\left(A_{2 \varepsilon_{0}} \cup \bar{\Omega}\right)$. By combining this with the fact that the function $F$ has compact support and is Lipschitz outside the set $A_{\varepsilon_{0}} \cup \bar{\Omega}$, we finally 
obtain (1) for a.e. $x, y$ in $\mathbf{R}^{n}$ e.g. with the function $c\left(g_{2}+F\right)$ substituted in place of $g$, where $c$ is a large enough constant. The estimate for $\|F\|_{L^{p}\left(\mathbf{R}^{n}\right)}$ is immediate and we obtain that $F \in M^{1, p}\left(\mathbf{R}^{n}\right)$ with suitable bounds for the norm.

Remark 22. The motivation for presenting proof of Theorem 20 above was to demonstrate how the coincidence of the Sobolev-Hardy spaces with the spaces $M^{1, p}$ can also be used to give simple and unified proofs of extension results, since the above argument works unchanged also for $p>1$. If one considers extension results just for the spaces $M^{1, p}(\Omega)$ it is possible to considerably weaken the conditions on $\Omega$ by replacing uniformity by a so called measure density property of the domain $\Omega$. This result (in the case $p=1$ ) is contained in [17].

\section{Extension of the Hardy inequality}

For a subdomain $\Omega \subset \mathbf{R}$ we denote by $W_{0}^{1, p}(\Omega)$ the closure of $C_{0}^{\infty}(\Omega)$ in the space $W^{1, p}(\Omega)$. It is well-known that if $\partial \Omega$ is regular enough, then the classical Hardy inequality

$$
\int_{\Omega}\left(\frac{|u(x)|}{d(x, \partial \Omega)}\right)^{p} d x \leq C(p, n, \Omega) \int_{\Omega}|D u(x)|^{p} d x
$$

holds for $p \in(1, \infty)$. In this case we say that $\Omega$ carries the $p$-Hardy inequality. We refer e.g. to [21] for conditions on $\Omega$ which ensure the validity of (14). On the other hand, it is well-known that the Hardy inequality fails for the space $W_{0}^{1,1}$. This fact is closely connected to $W^{1,1}\left(\mathbf{R}^{n}\right)$ and $M^{1,1}\left(\mathbf{R}^{n}\right)$ being different, as the following simple example shows.

Example 23. Assume that $h \in L^{1}(\mathbf{R})$ satisfies $\operatorname{supp}(h) \subset[-2,2], h \geq 0$, and $h$ is even. Define a function $u \in W^{1,1}(\mathbf{R})$ by setting $u(x)=\int_{0}^{x} h(t) d t$. Pick any $g \in D(u) \cap L^{1}(\mathbf{R})$. By symmetry we may assume that $g$ is even without changing the integrability properties of $g$. By considering the condition (4) with respect to points $x$ and $-x$ we deduce that

$$
\left|\frac{u(x)}{x}\right| \leq g(x) \text { for a.e } x>0 .
$$

Thus $u(x) / x$ is integrable in a neighbourhood of the origin, assuming that $g \in L^{1}$. Especially this holds if $u \in M^{1,1}(\mathbf{R})$. In this case, (15) can be viewed as an analog of the Hardy-inequality for the function $u$. However, in general (15) fails for $u \in$ $W^{1,1}(\mathbf{R})$, since the choice $h(x) \sim\left(|x| \log ^{2}(1 /|x|)\right)^{-1}$ near the origin leads to $u(x) / x \sim$ $(|x| \log (1 /|x|))^{-1}$ for small $x$.

We say that a bounded subdomain $\Omega \subset \mathbf{R}^{n}$ is Lipschitz if each point $x \in \partial \Omega$ has a regular neighbourhood $U$ in the sense that, after an isometric change of coordinates, we have

$$
U \cap \partial \Omega=\left\{(y, h(t)): y \in B(0, \delta) \cap \mathbf{R}^{n-1}\right\}
$$

where $\delta>0$ and the Lipschitz function $h: B(0, \delta) \cap \mathbf{R}^{n-1}$ may depend on $x$, but the Lipschitz constants of the functions $h$ are uniformly bounded. This enables us to define a local reflection $H$ on the neighbourhood $U \cap \Omega$. Thus, assuming that in the above coordinate system $\Omega$ lies locally below the graph of the function $h$, we define for $x=(y, t) \in U \cap \Omega$

$$
H(x)=(y, 2 h(y)-t) .
$$


The reflection map $H$ is a measure preserving bijection $H: U \cap \Omega \rightarrow H(U \cap \Omega)$. Morever, by shrinking the neighbourhood $U$ if needed, we may assume that $\sim \mid H(x)-$ $x \mid \leq c_{1} d(x, \partial \Omega)$, since $h$ Lipschitz.

The following result shows that the right counterpart of the Hardy inequality in the case $p \leq 1$ involves the Hardy-Sobolev spaces. It should be noted that the proof is almost trivial thanks to Theorem 1 .

Theorem 24. Let $\Omega$ be a bounded Lipschitz subdomain of $\mathbf{R}^{n}$ and $p \in(n /(n+1), 1]$. Then there is a finite constant $C=C(p, n, \Omega)$ such that any $\varphi \in C_{0}^{\infty}(\Omega)$ satisfies the inequality

$$
\int_{\Omega}\left(\frac{|u(x)|}{d(x, \partial \Omega)}\right)^{p} d x \leq C \sum_{j=1}^{n}\left\|D_{j} f\right\|_{H^{p}\left(\mathbf{R}^{n}\right)}^{p} .
$$

Proof. Assume that $\sum_{j=1}^{n}\left\|D_{j} u\right\|_{H^{p}\left(\mathbf{R}^{n}\right)}^{p}=1$. Choose a finite cover of of $\partial \Omega$ by regular neighbourhoods. Let $U$ be one of these neighbourhoods and assume that its intersection with the boundary $\partial \Omega$ has the representation (16). By Theorem 1 we may choose a function $g \in D(u)$ with $\|g\|_{L^{p}\left(\mathbf{R}^{n}\right)} \leq c$. We note that $u$ vanishes outside $\Omega$, whence we obtain for almost every $x \in U \cap \Omega$ that

$|u(x)|=|u(x)-u(H(x))| \leq|x-H(x)|(g(x)+g(H(x))) \leq c_{1} d(x, \partial \Omega)(g(x)+g(H(x))$.

Since $H$ is measure preserving we thus obtain that

$$
\int_{U \cap \Omega}\left(\frac{|u(x)|}{d(x, \partial \Omega)}\right)^{p} d x \leq\left(2 c_{1}\right)^{p} \int_{U \cap \Omega} g(x)^{p} d x \leq\left(2 c_{1}\right)^{p} c^{p} .
$$

The claim follows by summing over the chosen regular neighbourhoods, and applying the Poincare inequality to the uncovered part of the domain.

Remark 25. Another, even easier approach to the above result would be to use the obvious bi-Lipschitz invariance of the space $M^{1, p}\left(\mathbf{R}^{n}\right)$ in order to reduce the boundary locally to a piece of a hyperplane, in which case the reflection argument is trivial.

As our last observation we generalize the above result to a more extensive class of domains. For that end we recall certain definitions. Let $U \subset \mathbf{R}^{n}$ be a bounded open set and $p \in(0,1]$. The Hardy p-capacity of a compact subset $E \subset U$, relative to $U$, is defined by setting

$$
\operatorname{cap}_{H^{1, p}}(E ; U):=\inf \left\{\sum_{k=1}^{n}\left\|D_{k} \varphi\right\|_{H^{p}\left(\mathbf{R}^{n}\right)}^{p}: \varphi \in C_{0}^{\infty}(U), \varphi(x) \geq 1 \text { for } x \in E\right\} .
$$

It is easily verified that one may replace the condition $\varphi \in C_{0}^{\infty}(U)$ above by $\varphi \in$ $W_{0}^{\infty}(U)$, i.e. the class of compactly supported Lipschitz functions.

We need an analogue of [12, Proposition 3]. The proof is a slight modification of the original argument in case $p>1$.

Lemma 26. Let $q>n /(n+1)$. Assume that $B \subset \mathbf{R}^{n}$ is a ball with radius $r, K \subset B$ is compact with $\operatorname{cap}_{H^{1, q}}(K ; 2 B) \geq c r^{n-q}$. Then for every $u \in C_{0}^{\infty}\left(\mathbf{R}^{n}\right)$ satisfying $u_{\mid K}=0$, any $g \in D(u)$, and for any $x \in \bar{B}$ it holds that

$$
|u(x)| \leq r c(n, q)\left(M\left(g^{q}\right)(x)\right)^{1 / q} .
$$


Proof. For $k \geq 0$ denote $B_{k}=B\left(x, r 2^{-k}\right)$. The Poincare inequality, i.e. Proposition 5 yields that $\left|u_{2 B}-u_{B_{0}}\right| \lesssim r\left(M\left(g^{q}\right)(x)\right)^{1 / q}$ and $\left|u_{B_{k}}-u_{B_{k+1}}\right| \lesssim 2^{-k} r\left(M\left(g^{q}\right)(x)\right)^{1 / q}$. Since $u$ is continuous we may sum up these inequalities and obtain

$$
\left|u(x)-u_{2 B}\right| \lesssim r\left(M\left(g^{q}\right)(x)\right)^{1 / q} .
$$

In order to estimate $u_{2 B}$ we assume that $u_{2 B} \neq 0$. Define a cutoff function $h$ by setting $h(x)=1$ on $B, h(y)=1-2 \operatorname{dist}(x, B) / r$ for $x \in(3 / 2) B \backslash B$, and $h=0$ for $x \notin(3 / 2) B$. Set $v:=\left(u-u_{2 B}\right) h$. It is easily verified that $\widetilde{g} \in D(v)$, where

$$
\widetilde{g}:=\frac{2}{r}\left|u-u_{2 B}\right| \chi_{2 B}+g \chi_{2 B} .
$$

By $(q, q)$-Poincare, i.e. Proposition 5 combined with the Hölder-inequality, we have that $\|v\|_{L^{q}\left(\mathbf{R}^{n}\right)}^{q} \lesssim \int_{3 B} g^{q} d x$. Now $v / u_{2 B}$ is admissible for the definition of capacity, and we may apply Theorem 1 and the definition of capacity to estimate

$$
c r^{n-q} \leq \operatorname{cap}_{H^{1, q}}(K ; 2 B) \lesssim\left(u_{2 B}\right)^{-q} \int_{\mathbf{R}^{n}}|\widetilde{g}|^{q} d x \lesssim\left(u_{2 B}\right)^{-q} \int_{3 B}|g|^{q} d x .
$$

This yields an upper bound for $u_{2 B}$, which in combination with (19) finishes the proof.

Let $p \in(0,1]$. We call the complement of a domain $\Omega \subset \mathbf{R}^{n}$ uniformly $p$-fat if there is a constant $c>0$ such that for each $x \in \Omega^{c}$ and $r>0$ one has

$$
\operatorname{cap}_{H^{1, p}}\left(\Omega^{C} \cap B(x, r) ; B(x, 2 r)\right) \geq c r^{n-p} .
$$

Similarly, the complement $\Omega^{c}$ is thick in the sense of $s$ - Hausdorff content (here $s>0$ is arbitrary) if there is $c>0$ such that for each $x \in \Omega^{c}$ and $r>0$ one has

$$
\mathcal{H}_{\infty}^{s}\left(\Omega^{c} \cap B(x, r)\right) \geq c r^{s} .
$$

Here for any subset $E \subset \mathbf{R}^{n}$ the $s$-Hausdorff content of $E$ is defined as $\mathcal{H}_{\infty}^{s}(E):=$ $c(n, q) \inf \sum_{k=1}^{\infty} r_{k}^{s}$, where the infimum is taken over all denumerable coverings of $E$ by balls with radii $r_{k}, k \in \mathbf{N}$.

Theorem 27. Let $p \in(n /(n+1), 1]$. (i) Assume that $\Omega \subset \mathbf{R}^{n}$ is a bounded domain with uniformly $q$-fat complement for some $q<p$. Then the domain $\Omega$ carries the p-Hardy inequality (17).

(ii) Assume that the complement of $\Omega$ is thick in the sense of $(n-q)$-Hausdorff content with some $q<p$. Then the domain $\Omega$ carries the p-Hardy inequality (17).

Proof. (i) Let $u \in C_{0}^{\infty}(\Omega)$ and $g \in D(u)$. Fix $x \in \Omega$. Denote $r=d(x, \partial \Omega)$ and choose $z \in \partial \Omega$ with $d(x, z)=r$. We may apply Lemma 26 with the choice $B=B(z, r)$. The outcome is

$$
u(x) / d(x, \partial \Omega) \leq c\left(M\left(g^{q}\right)(x)\right)^{1 / q} .
$$

As $g^{q} \in L^{p / q}$, where $p / q>1$ we obtain the desired conclusion by the Hardy-Littlewood maximal theorem.

(ii) It is clearly enough to prove the following: given $n /(n+1)<q<p \leq 1$ and $C>0$ there is a constant $c=c(n, p, q)>0$ such that the condition $H_{\infty}^{n-q}(E) \geq C r^{n-q}$ implies that $\operatorname{cap}_{H^{1, p}}(E ; 2 B) \geq c r^{n-p}$. Here $B \subset \mathbf{R}^{n}$ is a ball and $E \subset \bar{B}$ is an arbitrary compact subset. Actually, since both the capacity and the Hausdorff content scale in the appropriete manner, it is enough to consider the case $r=1$. 
In order to prove the claim we thus assume that $B$ is a ball of radius one and $E \subset \bar{B}$ is compact with $H_{\infty}^{n-q}(E) \geq c_{0}$. Suppose that $u \in C_{0}^{\infty}(2 B)$ satisfies $u \geq 1$ on $E$ and $g \in D(u)$. Fix an arbitrary $x \in E$. Choose a ball $B_{0} \subset 3 B$ with radius 1 such that $B_{0} \cap 2 B=\emptyset$. Moreover, for $k \geq 1$ denote $B_{k}=2^{1-k} B$. By using again the Poincare inequality to compare mean values in the chosen balls we deduce that

$$
1=\left|u(x)-u_{B_{0}}\right| \leq c_{1}\left(\left(f_{3 B} g^{q} d x\right)^{1 / q}+2^{-k} \sum_{k=1}^{\infty}\left(f_{B_{k}} g^{q} d x\right)^{1 / q}\right) .
$$

Since $\sum_{k=0}^{\infty} 2^{(-1+q / p) k}=c_{2}<\infty$, we deduce that necessarily either $\left(f_{3 B} g^{q} d x\right)^{1 / q} \geq$ $1 /\left(c_{1} c_{2}\right)$ or there is an index $k \geq 1$ such that $\left(f_{B_{k}} g^{q} d x\right)^{1 / q} \geq 2^{k q / p} /\left(c_{1} c_{2}\right)$. In both cases, by the Hölder inequality, there is a constant $c_{3}=c_{3}(p, q, n)$ such that one of these balls, call it $\widetilde{B}$, has radius $\widetilde{r} \leq 3$, is centered at $x$ and satisfies

$$
\int_{\widetilde{B}} g^{p} d x \geq c_{3} \widetilde{r}^{n-q} .
$$

We select a ball $\widetilde{B}$ as above for each $x \in E$. By a standard covering argument we may select a disjoint subcollection $\mathcal{B}$ such that the corresponding 5 -times inflated balls cover $E$. By summing the inequality (23) over this collection we obtain

$$
\int_{\mathbf{R}^{n}} g^{p} d x \geq \sum_{\widetilde{B} \in \mathcal{B}} \int_{\widetilde{B}} g^{p} d x \geq c_{3} 5^{q-n} H_{\infty}^{n-q}(E) \geq c_{3} 5^{q-n} c_{0} .
$$

This concludes the proof of part (ii) of the Theorem.

Remark 28. The proof of part (ii) is insipired by [15]. In connection with the above result we point out the interesting paper [25] which connects the $p$-Hardy Sobolev capacity directly to the $(n-p)$-Hausdorff content. It should be also noted that in the case $p>1$ one may show the sufficiency of uniform $p$-fatness of $\Omega^{c}$ by employing the self-improving property of uniform $p$-fatness, due to J. Lewis [21], which approach appears not to work for $p \leq 1$. We plan to return to Hardy's inequality in more general domains and consider at the same time generalizations of the results of the present paper to non-Euclidean situations.

\section{References}

[1] R. A. Adams and J. J. F. Fournier: Sobolev spaces. Second edition. Academic Press 2003.

[2] P. Auscher, E. Russ, and P. Tchamitchian, Hardy Sobolev spaces on strongly Lipschitz domains of $\mathbf{R}^{n}$, J. Funct. Anal. 218 (2005), no. 1, 54-109.

[3] D.-C. Chang, S.G. Krantz, and E.M. Stein, $H^{p}$ theory on a smooth domain in $R^{N}$ and elliptic boundary value problems, J. Funct. Anal. 114 (1993), 286-347.

[4] D.-C. Chang, G. Dafni, and E.M. Stein, Hardy spaces, BMO, and boundary value problems for the Laplacian on a smooth domain in $\mathbf{R}^{n}$, Trans. Amer. Math. Soc. 351 (1999), 1605-1661.

[5] Y.-K. Cho, Strichartz conjecture on Hardy-Sobolev spaces, Colloq. Math. 103 (2005), 99-114.

[6] R. R. Coifman, A real variable characterization of $H^{p}$, Studia Math. 51 (1974), 269-274.

[7] R. DeVore and R. C. Sharpley, Maximal functions measuring smoothness. Mem. Amer. Math. Soc. 47 (1984), no. 293.

[8] C. Fefferman and E. M. Stein, $H^{p}$ spaces of several variables, Acta Math. 129 (1972), 137-193.

[9] A. Gatto, C. Segovia, and J. Jimnez, On the solution of the equation $\Delta^{m} F=f$ for $f \in H^{p}$. Conference on harmonic analysis in honor of Antoni Zygmund, Vol. I, II (Chicago, Ill., 1981), 394-415, 1983.

[10] D. Goldberg, A local version of Hardy spaces, Duke Math. J. 46 (1979), 27-42.

[11] P. Hajłasz, Sobolev spaces on an arbitrary metric space, Potential Anal. 5 (1996), 403-415. 
[12] _ Pointwise Hardy inequalities, Proc. Amer. Math. Soc. 127 (1999), 417-423.

[13] _ Sobolev spaces on metric-measure spaces, Heat kernels and analysis on manifolds, graphs, and metric spaces (Paris, 2002), 173-218, Contemp. Math., 338, Amer. Math. Soc., Providence, RI, 2003.

[14] _ A new characterization of the Sobolev space, Studia Math. 159 (2003), 263-275.

[15] P. Hajłasz and P. Koskela, Sobolev meets Poincaré, C. R. Acad. Sci. Paris Sr. I Math. 320 (1995), no. 10, 1211-1215.

[16] Sobolev met Poincaré, Mem. Amer. Math. Soc. 145 (2000).

[17] P. Hajłasz, P. Koskela, and H. Tuominen, Measure density and extendability of Sobolev functions, Rev. Mat. Iberoamericana (to appear).

[18] S. Janson, On functions with derivatives in $H^{1}$. IN: Harmonic analysis and partial differential equations (El Escorial, 1987), 193-201. Springer Lecture Notes in Math., 1384, 1989

[19] A. Jonsson, P. Sjögren, and H. Wallin, Hardy and Lipschotz spaces on subdomains of $R^{n}$, Studia Math 80 (1984), 2, 141-166.

[20] J. Kinnunen and O. Martio, Hardy's inequalities for Sobolev functions, Math. Res. Lett. 4 (1997), 489-500.

[21] J. Lewis, Uniformly fat sets. Trans. Amer. Math. Soc. 308 (1988), 177-196.

[22] A. Miyachi, $H^{p}$ spaces over open subsets of $R^{n}$, Studia Math 95 (1990), 205-228.

[23] _ Extension theorems for real variable Hardy and Hardy-Sobolev spaces. Harmonic analysis (Sendai 1990), 170-182, ICM-90 Satell. Conf. Proc., Springer, Tokyo 1991.

[24] _ Hardy-Sobolev spaces and maximal functions, J. Math. Soc. Japan 42 (1990), no. 1, 73-90.

[25] J. Orobitg, Spectral synthesis in spaces of functions with derivatives in $H^{1}$, Harmonic analysis and partial differential equations (El Escorial, 1987), 202-206. Springer Lecture Notes in Math., 1384, 1989.

[26] J. Peetre, New thoughts on Besov spaces. Duke Univ. Math. Ser, Duke University 1976.

[27] E. M. Stein, Harmonic Analysis. Princeton University Press 1993.

[28] R. Strichartz, $H^{p}$ Sobolev spaces, Colloq. Math. 60/61 (1990), 129-139.

[29] A. Torchinsky, Real-variable methods in Harmonic analysis. Academic Press 1986.

[30] H. Triebel, Theory of function spaces, Monographs in Math. 78 Birkhäuser, 1983.

DEPARTMENT, SCHOOL, MAILING LOCATION

Department of Mathematics and Statistics, University of Jyväskylä, P.O. Box 35

(MaD), FIN-40014 University of Jyvëskylä, Finland

E-mail address: pkoskela@maths.jyu.fi

Department of Mathematics and Statistics, University of Helsinki, P.O. Box 68 (Gustaf Hällstrmin kAtu 2B), FI-00014 University of Helsinki, Finland.

E-mail address: eero.saksman@helsinki.fi 